\title{
Féeries
}

Études sur le conte merveilleux, XVII $-\mathrm{XIXe}$ siècle

$17 \mid 2021$

Contes en couleur

\section{Serpents verts sur les rives de l'Elbe}

La couleur et l'expérience du merveilleux dans Le Petit Zachée surnommé Cinabre et Le Vase d'or d'E.T.A. Hoffmann

Green Snakes on the Banks of the Elbe. Color and the Experience of Wonder in E.T.A. Hoffmann's Little Zacchaeus Called Cinnabar and The Golden Pot

\section{Isabella Mattazzi}

\section{(2) OpenEdition}

Journals

Édition électronique

URL : https://journals.openedition.org/feeries/3790

DOI : $10.4000 /$ feeries. 3790

ISSN : $1957-7753$

Éditeur

UGA Éditions/Université Grenoble Alpes

Édition imprimée

ISBN : 978-2-37747-327-4

ISSN : 1766-2842

Référence électronique

Isabella Mattazzi, «Serpents verts sur les rives de l'Elbe », Féeries [En ligne], 17 | 2021, mis en ligne le

17 décembre 2021, consulté le 17 janvier 2023. URL : http://journals.openedition.org/feeries/3790 ;

DOI : https://doi.org/10.4000/feeries.3790

Ce document a été généré automatiquement le 17 janvier 2023.

Tous droits réservés 


\title{
Serpents verts sur les rives de l'Elbe
}

\author{
La couleur et l'expérience du merveilleux dans Le Petit Zachée \\ surnommé Cinabre et Le Vase d'or d'E.T.A. Hoffmann \\ Green Snakes on the Banks of the Elbe. Color and the Experience of Wonder in \\ E.T.A. Hoffmann's Little Zacchaeus Called Cinnabar and The Golden Pot
}

\author{
Isabella Mattazzi
}

\section{Dispositifs de vision}

1 L'écriture d'E.T.A. Hoffmann ${ }^{1}$ se fonde sur une véritable obsession de la vision. La critique contemporaine s'est souvent concentrée sur la présence généralisée d'instruments d'optique dans ses contes. Télescopes, microscopes, lunettes sont un thème constant qui traverse toute sa production et constitue un nœud structurant non seulement littéraire, mais phénoménologique tout court. Dans la perspective d'une réalité perpétuellement déchirée entre le caractère prosaïque du quotidien et les merveilles d'un monde parallèle régi par les logiques complexes de l'imaginaire, les instruments d'optique, véritables "dispositifs de vision ${ }^{2}$ ", semblent constituer un passage privilégié offrant la possibilité d'opérer par un changement rapide de regard un changement également radical de dimension ontologique (que ce changement mène alors aux hauteurs sublimes de la vérité poétique ou à l'abîme de la folie peu importe).

2 Ainsi, le visuel s'avère être un élément essentiel pour la narration hoffmannienne et pour comprendre les règles qui la régissent ${ }^{3}$. Hoffmann lui-même reconnaît à plusieurs reprises sa dette envers la peinture. Si sa biographie nous donne l'image d'un écrivainmusicien-compositeur et chef d'orchestre apprécié, dans les années de sa jeunesse à Könisberg il prend des leçons de dessin, et sa correspondance avec son ami Theodor Gottlieb Hippel démontre à quel point il a d'abord caressé l'idée (ensuite abandonnée) de devenir peintre ${ }^{4}$. À partir de sa première rencontre en 1798 avec la grande peinture européenne à la Gemälde Galerie de Dresde - un incontournable pour toute la Frühromantik - jusqu'aux après-midis passés à Bamberg à la Pommersfeldener Galerie, en passant par les nombreuses visites à l'Académie de Berlin, il laisse des traces 
largement documentées des tableaux qu'il a vus, et ses lettres à Hippel ${ }^{5}$, tout comme ses œuvres littéraires, résonnent de nombreux échos et références picturales ${ }^{6}$. Mais si alors son rapport à l'art semble être pleinement entré dans le canon de la réception critique contemporaine, il y a néanmoins un aspect de son écriture non encore exploré qui pourrait constituer une pièce supplémentaire pour reconstruire la phénoménologie de la vision hoffmannienne : la couleur comme élément descriptif pur et simple.

Dans les œuvres d'Hoffmann, la réalité et le merveilleux se déclinent selon une palette chromatique parfaitement définie. Les objets qui peuplent son écriture ne sont pas des éléments opaques, esquissés par la pointe sèche d'un mot en noir et blanc, mais vibrent de teintes, de tons, de dégradés. Les couleurs chez Hoffmann reviennent souvent avec les mêmes nuances identiques en créant des liens, des lignes souterraines qui vont d'un texte à l'autre comme s'il s'agissait de Leitmotive coloristes trop insistants pour ne pas suggérer une portée symbolique, ou du moins une importance structurante. Ce système de références apparaît plus qu'évident dans les contes de fées, un corpus de textes qui en l'espace productif d'une carrière de treize ans (depuis 1809, année de publication du Chevalier Gluck jusqu'à 1822, année de la mort de l'auteur) peut facilement être pris comme un noyau à part ${ }^{7}$. Le Petit Zachée surnommé Cinabre, Le Vase d'or, Maître Puce, La Princesse Brambilla présentent des caractéristiques thématiques et formelles homogènes, et on peut aisément reconnaître un certain air de famille entre eux dès les premières pages.

4 Dans le cas des contes de fées hoffmanniens, nous n'avons pas affaire au Märchen classique mais à une variante plus sophistiquée qui peut être inscrite, comme l'écrit Matteo Galli, dans le « Kunstmärchen, c'est-à-dire le conte de fées de maître, un conte de fées d'art où le nom Kunst qui précède le nom Märchen signifie certes art, artistique, mais aussi artificiel, artefact, tout comme Kunsthoff signifie plastique et Kunstleder simili cuir $^{8}$ ». Par rapport au conte de fées classique, le Kunstmärchen, tout en reconnaissant la valeur fondatrice de sa matrice populaire, la soumet à une action de problématisation substantielle, en la déformant, en jouant avec ses éléments constitutifs par des procédures de mise en abyme qui semblent atteindre chez Hoffmann des hauteurs presque postmodernes.

5 Avec ses interférences continues dans le texte, les clins d'œil au lecteur, les réflexions métalittéraires, les énoncés extradiégétiques, Hoffmann soumet sa matière narrative à des mécanismes constants de corrosion ironique qui fonctionnent comme un véritable agent agitateur en ce qui concerne la stabilité du pacte de lecture. Si, dans le conte de fées classique, le lecteur se retrouve dans le "merveilleux pur", adhérant avec une totale suspension of disbelief à tout ce qui est proposé dans l'histoire, dans les contes d'Hoffmann le merveilleux est confronté à une remise en question structurelle et constante de la vérité de son discours. "Je rêve, suis-je fou ou est-ce que tout est vrai ?», se demandent tout le temps les personnages. «Dois-je croire ce que je lis ou est-ce juste un monde en papier mâché mis en place par un auteur farceur?", se demande le lecteur' ${ }^{9}$. Le merveilleux dans le texte n'est jamais donné comme une condition première et absolue, mais doit se frayer un chemin dans une réalité qui lui est hostile, ou qui en tout cas ne semble jamais prête à le recevoir. L'indétermination spatio-temporelle de la formule "il était une fois dans un pays lointain» a été remplacée par le concret d'un quotidien bourgeois structurellement sourd à la voix enchanteresse de la magie et à la liberté illimitée de ses pouvoirs. Comme l'écrit Matteo Galli, «Hoffmann rejette les conventions, place les événements dans sa propre 
contemporanéité, dans un univers de serpents et de salamandres mais aussi d'archivistes et de vice-doyens, en tirant le maximum de profit poétique de la conflagration et de l'interrogation continue de ces deux mondes ${ }^{10} \%$. Et c'est précisément sur cette conflagration, ou plutôt sur ces entrelacements et contrastes perpétuels de deux univers inconciliables - réalité bourgeoise et merveilleux poétique - que le rôle de la couleur s'inscrit dans la syntaxe compositionnelle et symbolique d'Hoffmann.

\section{Le Petit Zachée surnommé Cinabre}

Dans Klein Zaches genannt Zinnober ${ }^{11}$ (Le Petit Zachée surnommé Cinabre), de 1819, la couleur semble occuper une place prépondérante dès le titre : le personnage de Zachée appelé Cinabre porte une référence chromatique dans son nom même, et il en est ainsi pour sa fée marraine Rosabelverde (en italien dans le texte, littéralement la fée « Rosebeau-vert »), et pour la jeune Candida (également en italien dans le texte). L'histoire s'ouvre sur une scène de sortilège exécutée par Rosabelverde sur un enfant aux traits horribles, une sorte de tubercule à pattes, "une petite mandragore" (ein klein Alräunchen $\left.{ }^{12}\right)$. La fée, rencontrant dans une clairière une pauvre paysanne visiblement accablée d'avoir à côté d'elle ce petit monstre incapable de faire quoi que ce soit, décide de l'aider et caresse les cheveux de l'enfant, en lui donnant, par ce geste, le pouvoir d'absorber les qualités positives de quiconque est à côté de lui. En d'autres termes, chaque fois que Zachée s'approche d'un beau jeune homme, lui-même paraîtra beau aux yeux de tous. Chaque fois qu'il s'assoit à côté d'un poète ou d'un brillant causeur, les vers du poète, les plaisanteries intelligentes du causeur sortiront, pour le public, de sa bouche et non de celui à qui ils appartiennent vraiment. La force du don magique de la fée réside dans "trois cheveux brillants couleur du feu " (drei feuerfarbglänzenden Haaren $^{13}$ ) sur le dessus de la tête de l'enfant, cachés jalousement à tous les yeux sous une épaisse masse de boucles et arrachés à la fin de l'histoire par l'étudiant Balthasar, le seul capable de percevoir la vérité derrière le sortilège et de démasquer la tromperie. La couleur rouge semble donc appartenir au petit Zachée comme un trait non seulement descriptif mais aussi structurant, puisque l'identité magique du personnage est totalement contenue dans le rouge. En l'absence de rouge, Zachée est une créature inutile et monstrueuse, un " petit poucet » (Däumling $\left.{ }^{14}\right)$. Immergé dans le rouge, Zachée peut devenir celui qu'il veut, récolter les honneurs sans les mériter, atteindre le poste convoité de premier ministre et se fiancer à la belle Candida.

7 L'élément chromatique, le rouge de ses trois cheveux enchantés, pénètre largement dans l'identité du personnage jusqu'à ce qu'il en soit complètement teint. Ses vêtements dorénavant seront rouges ( $u$ un habit écarlate brodé15 $)$ ), un pigment rouge, le cinabre, deviendra son nom. Après la scène d'introduction de la clairière, nous retrouvons en fait Zachée, désormais adulte, au moment de son entrée dans la ville de Kerepes où vit le jeune étudiant Balthasar. Hoffmann, sans nous donner aucune explication sur l'origine du surnom, se borne à annoncer par la voix du professeur Mosch Terpin : "Permettez-moi, mesdames et messieurs, de vous présenter un jeune homme doué des qualités les plus rares, et qui saura sans peine gagner votre estime et votre bienveillance. C'est le jeune seigneur Cinabre: il n'est pas arrivé que d'hier à notre université et se destine à l'étude du droit ${ }^{16}$. » Et à partir de là, «Cinabre » sera le premier terme identifiant le personnage. 
8 Le cinabre (sulfure de mercure) est un minéral appartenant à la classe des sulfures. Sa couleur rouge caractéristique a été utilisée comme pigment, depuis l'Antiquité, par les peintres et les miniaturistes et a eu une diffusion si large dans le domaine artistique qu'elle est devenue ni plus ni moins qu'un synonyme de rouge ${ }^{17}$. Les nuances du pigment sont variées : elles vont d'un rouge orangé (appelé cinabre hollandais) à un rouge laque (le vermillon chinois), en passant par un rouge presque violacé très semblable au rouge de cadmium pourpre. Produit artificiellement par les alchimistes qui ont commencé à synthétiser du sulfure de mercure à partir de soufre et de mercure, le cinabre a une odeur sulfureuse piquante qui en fait un métal historiquement lié au démoniaque et à tout ce qui le connote ${ }^{18}$. Hoffmann a certainement dû prendre en compte ces caractéristiques culturelles du cinabre, même si le personnage de Zachée dans le texte ne présente aucune nuance inquiétante ${ }^{19}$. Le plus grand péché de Cinabre est sa vanité, ou plutôt son incapacité à s'élever au-dessus « de sa grossièreté, de sa sottise, et de sa rudesse ${ }^{20} »$, et sa véritable vocation est celle d'un personnage de comédie, comme en témoigne sa sortie de scène au moment où il finit noyé dans son propre pot de chambre ${ }^{21}$. Mais au-delà de sa nature plus ou moins malveillante, l'élément le plus intéressant de Cinabre semble être son adhésion identitaire totale à une nuance chromatique. À la perte de sa couleur, quand ses cheveux roux lui seront arrachés, il ne pourra rien faire d'autre que disparaître de l'histoire, s'effaçant littéralement dans la pâleur de la mort ${ }^{22}$.

9 L'identité de la fée Rosabelverde coïncide également avec la couleur de son nom. Dans une perspective préromantique qui voit dans la communion originelle entre homme et nature un idéal harmonique perdu et en même temps toujours présent en tant qu'horizon de modélisation à atteindre, les personnages magiques d'Hoffmann sont presque toujours associés à une idée de merveilleux naturel. La fée Rosabelverde a le pouvoir de faire pousser les plus belles roses autour d'elle (nomen omen), elle parle aux oiseaux et est entourée de cygnes et de paons qui lui sont fidèles. Le vert qui caractérise son nom est donc le vert de la forêt, des prairies, d'une nature idyllique qui pour Hoffmann coïncide toujours avec l'idée d'un monde libre de toute forme de rationalisation et d'utilitarisme économique de matrice bourgeoise. Rosabelverde, comme tous les personnages qui appartiennent chez Hoffmann à une dimension magique, doit cependant défendre son droit d'exister dans une lutte sans quartier pour revendiquer son identité23. Afin de survivre dans une société hostile qui ne peut pas et ne veut pas reconnaître la complexité du réel, elle doit constamment atténuer la lumière de son regard, adoucir le pouvoir de ses dons, en cachant sa nature féerique derrière l'identité fictive de $\mathrm{M}^{\text {Ile }}$ von Rosenschön (littéralement " $\mathrm{M}^{\text {lle }}$ Roses-beau »), respectable célibataire du pensionnat de Kerepes. Dans le passage de Rosabelverde à Rosenschön, dans ce qui est une abdication (quoique temporaire) du merveilleux à la dimension apaisante d'un quotidien domestiqué, il est impossible de ne pas remarquer un changement évident de caractère chromatique. $\mathrm{M}^{\text {lle }}$ von Rosenschön (Roses-beau), abandonnant sa propre identité de fée Rosabelverde (Rose-beau-vert), de fait a perdu le vert $^{24}$.

10 À une soustraction du merveilleux dans le monde semble correspondre ainsi une soustraction de couleur, exactement comme en sens inverse une addition de merveille implique - c'est le cas de Zachée devenu Cinabre après le sortilège - aussi, forcément, une addition chromatique. En d'autres termes, la couleur fonctionne dans le texte comme un indicateur, un gradient de merveille : à une intensité plus ou moins grande 
du merveilleux coïncide, de manière directement proportionnelle, une saturation chromatique plus ou moins grande sur la page.

11 Le monde magique dans Le Petit Zachée se caractérise en fait par une surabondance et une palette de couleurs totalement absentes de la dimension normée de la vie quotidienne. Les oiseaux dont Rosabelverde s'entoure ont « un plumage étincelant et diapré » (mit buntem glänzenden Gefieder ${ }^{25}$ ), les unicornes qui conduisent la calèche du magicien Prosper Alpanus sont «blancs comme neige » (schneeweiß $\left.e^{26}\right)$, les scarabées et faisans qui les accompagnent sont d'or et d'argent, et les arbres du jardin du magicien ont des « troncs luisants et feuillage d'émeraude » (glänzenden Stämmen und smaragdenen Blättern ${ }^{27}$ ). Le même Alpanus « est vêtu d'une longue robe à l'indienne, de couleur ocre, et chaussé de petites bottines rouges à lacets dont on ne savait trop si elles étaient garnies d'une fourrure aux richesses couleurs ou d'un éclatant plumage d'oiseau ${ }^{28}$ » et si l'identité de $\mathrm{M}^{\text {lle }}$ von Rosenschön trouve protection et abri dans une très bourgeoise robe noire (c'est-à-dire dans une absence totale de couleur), la fée Rosabelverde est au contraire une émanation scintillante de blanc et de rouge :

On annonça que la chanoinesse von Rosenschön désirait parler à monsieur le docteur.

- Bien volontiers! répondit Prosper Alpanus. Et la dame entra. Elle portait une longue robe noire et était enveloppée de voiles à la manière d'une matrone. Frappé d'un étrange pressentiment, Prosper Alpanus prit sa canne et dirigea sur la chanoinesse les rayons étincelants du pommeau de cristal. Ce fut alors comme de vifs éclairs tressaillaient et bruissaient autour d'elle et elle apparut, vêtue d'une tunique blanche et transparente, les épaules munies de brillantes ailes de libellule et les cheveux entrelacés de roses blanches et rouges. - Tiens, tiens! murmura le docteur. Puis il dissimula la canne sous sa robe de chambre et la dame reprit aussitôt son premier costume ${ }^{29}$.

La couleur chez Hoffmann - tout comme la musique, la métamorphose, la synesthésie - relève du niveau du merveilleux (das Wunderbare), en venant ainsi se placer directement au centre exact de sa poétique. Lorsque le passage entre quotidien et dimension féerique est ouvert et qu'une nature au sens novalisien (c'est-à-dire comme un hiéroglyphe d'une langue perdue que seuls quelques privilégiés peuvent encore comprendre) dissout finalement son énigme, alors la couleur brille sur le monde en parfaite harmonie entre sujet sensible et objet.

\section{Le Vase d'or}

Dans Le Vase d'or (Der goldne Topf ${ }^{30}$ ), conte écrit en 1814, également structuré par un dédoublement constant entre réalité quotidienne et monde magique, cette fonction chromatique spécifique du discours apparaît encore plus évidente. Dans ce cas, l'environnement qui sert de toile de fond aux événements est la ville de Dresde, mentionnée dans le texte dans ses éléments urbains les plus immédiatement reconnaissables pour un lecteur de l'époque : les bains de Link, le marché de la Porte Noire, les rives de l'Elbe... En particulier, les rives de l'Elbe constituent un point névralgique pour la construction de l'histoire, puisque c'est précisément là que, à quelques pages du début du texte, l'étudiant Anselme est confronté à une déchirure soudaine de la surface du monde et à l'apparition d'une nouvelle possibilité de structuration de la réalité, c'est-à-dire encore une fois à la structuration d'un nouveau paradigme perceptif et à une palette chromatique totalement renouvelée : 
Mais, au même instant, il entendit au-dessus de sa tête l'accord de trois clochettes de cristal ; ayant levé ses yeux il aperçut trois petits serpents diaprés de vert et d'or: enroulés autour des branches ils tendaient leurs têtes effilées vers le soleil couchant. [...] En même temps, les petits serpents coulaient et s'enlaçaient entre les feuilles et les branches; la rapidité de leurs mouvements était si grande qu'on croyait voir le sureau étinceler de mille émeraudes au travers de sa ramure sombre. "C'est sans doute le soleil couchant, pensait maintenait Anselme, qui miroite dans le feuillage... » Mais il entendit à nouveau le tintement des clochettes et aperçut un serpent qui abaissait vers lui sa petite gueule. Une décharge électrique ne l'eût pas fait frémir davantage et il sentit un tremblement au plus profond de lui-même ; mais ayant levé ses regards éperdus, il reçut le choc de deux yeux splendides au bleu profond [...]. Tout enfiévré de désir, il rivait ses regards sur ces yeux adorables; il entendait à nouveau, et plus fort, les accords ravissants des cloches de cristal et il se trouva soudain au milieu d'un ruissellement d'émeraudes qui l'encerclaient de mille flammes, où se jouaient d'étincelants fils d'or ${ }^{31}$.

Le vert de la peau des serpents et le bleu de leur regard, l'or des branches au coucher du soleil, avec leur puissance de timbre capable de créer un véritable choc perceptif (« une décharge électrique ») indiquent concrètement que le seuil a été franchi. La dimension magique surgit immédiatement dans la page, déployant une structure de dérèglement sensible, ou plutôt un espace dans lequel les règles de perception de l'objet ne sont pas les mêmes que dans le monde réel. En premier lieu, les couleurs apparaissent comme les vecteurs d'une augmentation sensorielle hors du commun. Dans le monde merveilleux hoffmannien, toute référence chromatique est toujours caractérisée par un surplus de lumière : étincelant (funkelnd), diapré (bunt), lumineux (leuchtend), luisant (glänzend), éblouissant (blendend) sont des adjectifs récurrents d'un texte à l'autre. Deuxièmement, l'utilisation massive de la synesthésie dans les stratégies descriptives du merveilleux hoffmannien place la couleur au cœur d'une polysensorialité rayonnante qui voit dans la coïncidence de sonorités et couleurs son résultat le plus élevé. Hoffmann connaît bien la théorie de Louis-Bertrand Castel sur la nature commune de la lumière et $\mathrm{du}$ son $^{32}$, et dans l'un de ses textes mineurs, Une lettre du maître de chapelle Johannes Kreisler (1819), il cite son clavecin oculaire, même si c'est pour s'en distancer ${ }^{33}$ :

Ce n'est pas la couleur verte, c'est le bois, avec la beauté gracieuse de son feuillage qui engendre un frisson et une douce mélancolie dans notre poitrine. Le bleu profond du ciel commencera bientôt à nous paraître vide et triste quand il n'y aura pas de nuages s'élevant en myriades d'images changeantes. Appliquez cela à l'art et rendez-vous compte que vous serez bientôt fatigué de voir les plus belles couleurs sans aucune forme, et combien brièvement la vue en serait chatouillée. Pensez au ridicule clavecin oculaire du Père Castel ! - Et il en va de même en musique. Le ton n'affectera profondément l'âme que lorsqu'il se sera transformé en mélodie ou en harmonie, bref, en musique ${ }^{34}$.

14 Le passage de la lettre de Johannes Kreisler sur la relation entre couleur et dessin clarifie parfaitement la position d'Hoffmann non seulement en tant que peintre ou musicien, mais aussi en tant qu'écrivain. Si, sur la toile, le timbre chromatique sans le dessin ne peut provoquer que des sensations superficielles, et si la couleur du son sans l'harmonie ou la mélodie ne peut pas s'appeler exactement musique, nécessairement, sur la page, la narration (« les myriades d'images changeantes ») occupera une place privilégiée dans le processus de production de sens. Dans Le Vase d'or, la couleur accompagne la narration d'un niveau de réalité à un autre en s'allumant et s'éteignant comme un interrupteur et, tout comme un interrupteur, elle ne fonctionne qu'en présence d'un sujet capable de l'activer. La donnée chromatique semble être moins une 
qualité de la réalité qu'une qualité de l'œil de celui qui la contemple. Il faut observer que dans le texte la tombée de la nuit qui met fin à la vision d'Anselme et qui fait sombrer les rives de l'Elbe dans l'obscurité est exprimée par la voix d'un passant, c'està-dire un sujet bourgeois qui, par nature, est incapable de comprendre et donc de « voir» un monde différent du sien («Eh, mon bon monsieur! Dit-il, inutile de vous lamenter ainsi dans le noir! Et n'importunez pas les honnêtes gens, quand tout votre mal est d'avoir plus que de raison $\left.{ }^{35} ! »\right)$. Anselme lui-même, dès lors qu'il se met à douter de l'existence du monde magique, perçoit les couleurs différemment. Si le cabinet du magicien Lindhorst, prince des salamandres, était jusqu'alors pour lui un lieu vital d'émerveillement et de régénération, lorsqu'Anselme commence à vaciller dans son amour pour le serpent vert et dans sa foi en la dimension magique parallèle, cet espace se transforme soudain (à ses yeux) en une baraque de foire :

La chambre bleue, elle aussi, était toute changée et il n'arrivait pas à comprendre comment ce bleu criard, ce faux doré des palmiers, et ces palmes en toc biscornues avaient pu lui plaire ${ }^{36}$.

La dégradation chromatique des objets dans la bibliothèque de Lindhorst, le glissement ruineux du bleu-or de " étincelante » et «splendide » à " criard » et « faux ", ainsi que l'obscurcissement soudain de la campagne autour de l'Elbe montrent comment la couleur n'est pas un élément inhérent à la nature de l'objet, mais suit les fluctuations du regard (et de la pensée) du sujet.

En cela, Hoffmann adopte les vues sur la nature de la lumière de J. W. Goethe qui, dans son ouvrage Zur Farbenlehre (Traité des couleurs) de 1810, a examiné le débat scientifique sur la couleur à partir de sa perspective personnelle des sciences naturelles. En controverse ouverte avec I. Newton, Goethe voit la nature comme l'expression vitale d'un Esprit (au sens romantique), et non comme un ensemble de phénomènes réductibles à des enchaînements rigides de causes et d'effets. La phénoménologie de la couleur de Goethe doit donc être porteuse d'informations qualitatives et non seulement quantitatives, en incluant aussi les données esthétiques et spirituelles du phénomène de la couleur, et surtout en les inscrivant dans une réflexion plus large sur le rapport entre l'Homme et la Nature. En d'autres termes, dans le Traité des couleurs, ce n'est pas tant la lumière en tant que telle qui est au centre du discours mais l'œil, et avec lui le mécanisme de la vision et la spiritualité de l'âme de l'observateur ${ }^{37}$.

Pour Hoffmann, faire l'expérience du merveilleux signifie regarder le monde à travers d'autres yeux dans un processus de transformation mutuelle qui voit le sujet et la réalité qui l'entoure changer en même temps. Au moment où Balthasar ou Anselme touchent pour la première fois la profondeur de leur propre âme poétique, le monde se révèle dans sa vérité la plus radicale qui, pour Hoffmann, est toujours une vérité de mélange et de métamorphose : des serviteurs aux livrées multicolores s'avèrent être des toucans ou des grenouilles, des capes flottantes prennent leur envol alors que des vautours à grandes ailes, des archivistes, des médecins, des jeunes femmes sans mari montrent leur vrai visage de sorciers, de fées, de princes salamandres.

\section{Chromatismes merveilleux}

Mais peut-on parler d'une qualité propre aux couleurs du monde enchanté ? En quoi les couleurs de la dimension magique diffèrent-elles des nuances chromatiques qui entourent et accompagnent notre vie quotidienne? 

thèmes récurrents, toujours représentés par la même couleur. La nature - que nous avons vue s'articuler dans la peau des serpents, dans les feuilles du sureau, dans le nom de la fée Rosabelverde - est toujours verte avec des nuances d'émeraude. Le regard féminin, avec son pouvoir hypnotique et fascinant, est au contraire bleu. Alors que ce qui est pour Hoffmann une sorte de mythe fondateur de son cosmos littéraire (mythe présent entre autres dans Le Vase d'or et Le Petit Zachée), à savoir l'amour d'un princemagicien pour une princesse-fleur qui en un temps archaïque a donné origine et vie au monde enchanté, a comme trait chromatique dominant le rouge pourpre (purpur) de la fleur ${ }^{38}$.

La triade vert-bleu-pourpre s'inscrit parfaitement dans le paradigme chromatique du romantisme allemand. Si le vert coïncide avec l'idée d'une nature libérée de toute logique utilitaire et si le bleu - pour toute une génération d'écrivains formés sur le modèle novalisien - est la couleur de la pensée poétique ${ }^{39}$, le pourpre est défini par Goethe comme la couleur par excellence car elle « contient toutes les autres couleurs en partie $a c t u$, en partie potentia $a^{40} »$. Le pourpre-vert-bleu est donc l'axe chromatique privilégié pour une expérience sensorielle de correspondance absolue entre l'homme et le monde, ce sont les couleurs à travers lesquelles on peut faire coïncider son expérience intérieure, un Erlebnis mystique et esthétique, avec une réalité qui n'est plus perçue comme étrangère et hostile mais finalement comme harmonieuse et apaisée.

21 La prédominance de certains tons sur d'autres n'est cependant pas le seul élément permettant de caractériser le monde magique. Les objets du monde magique sont souvent faits d'or, d'argent et de pierres précieuses, un détail qui sert certainement à connoter la richesse matérielle et spirituelle de ceux qui possèdent ces objets, mais qui d'un point de vue strictement visuel implique un surplus de luminosité. L'or, l'argent, les émeraudes et les diamants sont des corps réfléchissants, leurs surfaces polies sont des agents de réfraction et de propagation de la lumière, et même en dehors des teintes naturellement brillantes comme l'or et l'argent, toute la palette du monde magique est toujours caractérisée par des adjectifs qui dénotent un fort renforcement de la qualité lumineuse. En substance, dans le monde magique, le bleu semble être encore plus profond et brillant, le rouge est toujours ardent et le blanc est d'une clarté aveuglante. Cette augmentation lumineuse constante de la couleur dans la dimension magique du récit en fait une sorte de variante de ce que l'on pourrait appeler l'obsession par excellence de la fiction hoffmannienne : les instruments d'optique.

lunettes, les télescopes, les lentilles ne sont pas seulement des outils qui affinent le regard, mais aussi des surfaces réfléchissantes. Leur fonction d'objets-ponts entre les deux dimensions de l'existence n'est pas seulement donnée par leur capacité structurelle à déformer le regard et à nous faire voir des portions de réalité jamais imaginées, mais aussi par leur pouvoir de réfléchir la lumière et de frapper nos yeux - tout comme les couleurs - avec une force peu commune. Qu'il s'agisse d'instruments optiques ou de couleurs chatoyantes, le résultat est toujours le même: un choc perceptif qui conduit inévitablement à une modification substantielle des paramètres de l'interaction sensible avec la réalité (et à ce que nous pourrions définir aujourd'hui comme une altération conséquente de l'équilibre psychique du sujet ${ }^{41}$ ). Les lunettes étincelantes que l'opticien Coppelius pose sur la table dans L'Homme au sable (1817) ne semblent pas si éloignées de l'anneau du magicien Lindhorst ou du pommeau de la 
canne d'Alpanus, capables d'émettre des rayons lumineux, ni de l'éclat translucide de la peau reptilienne de Serpentina :

Mais en moins d'un instant, Coppola s'était débarrassé de ses baromètres ; il mit les mains dans les larges basques de son habit, il en tira des lunettes et des conserves qu'il posa sur la table. - « Eh bien donc, eh bien, des lounettes, des lounettes pour mettre sul naso, voilà mes yeux à moi, - belli occhi, Signor! » Et il sortait lunettes sur lunettes, si bien que toute la table commença à rayonner et à scintiller d'une singulière façon. Nathanael voyait des milliers d'yeux croiser sur lui leurs regards et s'agiter convulsivement, mais sans pouvoir détourner sa vue de cet aspect; et Coppola déposait toujours plus de lunettes sur la table, et de nouveaux yeux étincelants lançaient des éclairs de plus en plus redoutables sur Nathanael, qui sentait leurs rayons d'un rouge de sang pénétrer ardemment dans sa poitrine ${ }^{42}$.

On connaît bien l'intérêt d'Hoffmann pour les études qui, entre le XVIII et le XIX siècle, ont animé le débat médico-scientifique sur l'(hyper)sensibilité. Dans les premières années de sa production littéraire, Hoffmann a manifesté à plusieurs reprises son vif intérêt pour le mesmérisme (Le Magnétiseur de 1814 est un récit entièrement consacré à ce thème), mais il s'en est ensuite progressivement éloigné après avoir reconnu son caractère impressionniste et pseudo-scientifique. Ce n'est pas un hasard si Prosper Alpanus, un personnage du Petit Zachée à la fonction évidemment thérapeutique et salvatrice (il est médecin-magicien), apparaît pour la première fois à Balthasar à bord d'un carrosse-glasharmonica (encore une fois étincelant de lumière et de couleurs), c'est-à-dire dans la transformation féerique d'un instrument de musique - l'harmonica de verre mis au point par Benjamin Franklin en 1761 - utilisé par F.-A. Mesmer pour induire la transe hypnotique chez ses patients ${ }^{43}$. Mais au-delà de la position d'Hoffmann sur les différents paradigmes qui, entre le XVIII et le $\mathrm{XIX}^{\mathrm{e}}$ siècle, se disputent le champ $\mathrm{du}$ traitement de ce qui sera défini ensuite par J.-M. Charcot comme des maladies neurologiques, reste inchangée la centralité de la couleur au sein de ce laboratoire expérimental qu'est en fait l'écriture hoffmannienne ${ }^{44}$. Si dans sa conception du dualisme de l'être, dans la scission continue des lignes de la narration et de l'existence, Hoffmann met en jeu les limites et les possibilités de notre sensibilité et de notre statut d'êtres humains tout court, la couleur dans ce jeu semble avoir un rôle certainement décisif.

\section{NOTES}

1. Pour l'original allemand des textes d'E.T.A. Hoffmann utilisés dans cet essai, on fait référence à l'édition des œuvres complètes en six volumes éditée par G. Allrogghen, H. Steinecke et F. Auhuber: E.T.A.Hoffmann, Sämtliche Werke in sechs Bänden, Deutscher Klassiker Verlag, Francfort, 2003.

Pour ce qui concerne la traduction française des contes analysés, on fera référence aux éditions suivantes :

E.T.A. Hoffmann, L'Homme au sable, dans Contes nocturnes, trad. A. Montandon, Paris, Classiques Garnier, 2011. 
E.T.A. Hoffmann, Le Petit Zachée surnommé Cinabre, trad. M. Laval, Paris, Éditions Phébus, 1980.

E.T.A. Hoffmann, Le Vase d'or, trad. A. Espiau de La Maëstre, Paris, Gallimard, 1964.

2. Le terme est utilisé par Michele Cometa dans M. Cometa et A. Montandon, Vedere. Lo sguardo di E.T.A. Hoffmann, Palerme, :duepunti edizioni, 2009.

3. Le célèbre essai de Sigmund Freud sur L'Homme au sable inaugure au début du $\mathrm{xx}^{\mathrm{e}}$ siècle une bibliographie presque sans fin sur le regard dans Hoffmann, démontrant la vitalité et l'importance du thème scopique dans son œuvre.

4. Hoffmann, en revanche, a continué à dessiner tout au long de sa vie, parsemant ses textes autographes de croquis dans les marges, de caricatures, de portraits d'amis ou de personnages inventés, véritables contrepoints visuels à la parole littéraire. Dans l'une de ses esquisses, une aquarelle de 1815 intitulée Le Maître de chapelle Johannes Kreisler en habit de maison dessiné d'après nature par Erasmus Spikher, Hoffmann se représente sous la forme de son alter ego musical, Johannes Kreisler (protagoniste du Chat Murr et de Kreisleriana), et déclare que le dessin est l'œuvre d'Erasmus Spikher, le peintre sans reflet du récit La Nuit du Nouvel An: en d'autres termes, une revendication manifeste, sous forme d'aquarelle, de sa triple identité musicale, visuelle et littéraire.

5. Pour la correspondance avec Theodor Gottlieb Hippel, voir E.T.A. Hoffmann, Sämtliche Werke in sechs Bänden - Band 1: Frühe Prosa. Briefe. Tagebücher. Libretti. Juristische Schrift. Werke 1794-1813, Deutscher Klassiker Verlag, Francfort, 2003, p. 11-147.

6. L'étude de Michele Cometa, Descrizione e desiderio. I quadri viventi di E.T.A. Hoffmann (Rome, Meltemi, 2005) fournit une cartographie détaillée des nombreuses ekphrasis contenues dans les textes d'Hoffmann, permettant ainsi de reconstituer fidèlement - par la comparaison précise entre texte et image - ce qu'a été la formation artistique de l'écrivain.

7. Il existe sept contes de fées hoffmanniens : Der goldne Topf. Ein Märchen aus der neuen Zeit (Le Vase d'or. Conte des temps modernes, 1814), Nussknaker und Mausekönig (Casse-Noisette et le roi des souris, 1816), Das fremde Kind (L'enfant étranger, 1817), Die Königsbraut. Ein nach der Natur entworfenes Märchen (La fiancée du roi. Un conte de fées conçu à partir de la Nature, 1821), Klein Zaches gennant Zinnober. Ein Märchen (Le Petit Zachée surnommé Cinabre. Conte, 1819), Prinzessin Brambilla. Ein Capriccio nach Jakob Callot (Princesse Brambilla. Un caprice dans la manière de Jacques Callot, 1820), Meister Floh. Ein Märchen in sieben Abenteuern zweier Freunde (Maître Puce. Conte en sept aventures survenues à deux amis, 1822).

Sur ces sept, quatre portent le terme Märchen en sous-titre et deux (Nussknaker und Mausekönig et Das fremde Kind) sont contenus dans le recueil de contes pour enfants Kinder-Märchen (1816) qu'Hoffmann a publié avec Friedrich de la Motte Fouqué et Carl Wilhelm Salice-Contessa.

8. M. Galli, «Il muscolo della fiaba », introduction à E.T.A. Hoffmann, Fiabe, Rome, L'Orma, 2014, p. XXVII (ma traduction).

9. Si l'on voulait utiliser les catégories todoroviennes, on pourrait dire que les contes d'Hoffmann semblent appartenir davantage au genre fantastique qu'au merveilleux.

10. M. Galli, ouvr. cité, p. XXVI.

11. Klein Zaches gennant Zinnober fait partie du troisième volume (Band 3: Nachtstücke/Klein Zaches/ Prinzessin Brambilla. Werke 1816-1820) de l'édition intégrale des œuvres d'E.T.A. Hoffmann (voir note 1) à laquelle on fait référence pour les citations en allemand du conte.

12. E.T.A. Hoffmann, Klein Zaches gennant Zinnober, éd. citée, p. 535.

13. Dans le texte, les trois cheveux magiques sont définis «brillants couleur du feu» (feuerfarbglänzenden Haare, p. 618 de l'édition allemande citée), «couleur du feu » (feuerfarbnen Haare, p. 618), ou tout simplement « roux» (roten Haare, p. 618). Quelquefois Hoffmann parle non pas de trois cheveux mais d'une raie des cheveux, elle aussi « couleur du feu » (feuerfarb glänzende Streif, p. 601) ou « rousse » (ein roter Streif, p. 639).

14. La référence à Perrault dans la présentation de Zachée sous le nom de Däumling (c'est-à-dire Petit poucet) n'est pas accidentelle, mais résonne comme une véritable revendication 
d'appartenance au genre du conte de fées (le terme revient neuf fois dans le texte). De même, les lourdes bottes dans lesquelles Zachée trébuche à plusieurs reprises dans la scène de son arrivée à Kerepes ne peuvent que rappeler les bottes du Chat botté (Ludwig Tieck en a écrit une version en 1797 sous la forme d'un conte théâtral) ou les bottes de Sept lieues (mentionnées dans un texte de 1814 qu'Hoffmann connaît bien, à savoir La Merveilleuse histoire de Peter Schlemihl d'Adalbert von Chamisso). Même la forte ressemblance sonore entre les localités de Kerepes (Le Petit Zachée genannt Zinnober) et de Carabas (Le Chat botté) ne peut que souligner la volonté d'Hoffmann de jouer sur le même registre par rapport au corpus des contes de Perrault qui circulait largement en Allemagne dans ces années-là.

15. Le terme allemand pour la couleur du vêtement est scharlachroten : «In dem mit hundert Kerzen erleuchteten Saal stand der kleine Zinnober im scharlachroten gestickten Kleide [...]. » (p. 626 de l'édition allemande citée).

16. E.T.A. Hoffmann, Le Petit Zachée surnommé Cinabre, éd. citée, p. 59.

17. P. Travaglio, «Una piccola storia del cinabro tra antichità e Rinascimento », Effetto Arte, $\mathrm{n}^{\circ} 1,2016$, p. 123-127.

Pour l'utilisation des pigments, voir R. Falcinelli, Cromorama. Come il colore ha cambiato il nostro sguardo, Turin, Einaudi, 2017. Sur l'histoire de l'utilisation de la couleur dans les textes scientifiques entre le XVIII ${ }^{\mathrm{e}}$ et le $\mathrm{XIX}^{\mathrm{e}}$ siècle, voir G. Maës, "De Dezallier d'Argenville à Darwin : la question de la couleur dans la représentation des minéraux», dans N. Vuillemin et E. Dueck (dir.), Entre l'œil et le monde. Dispositifs d'une nouvelle épistémologie visuelle dans les sciences de la nature (1740-1840), Éditions Épistémocritique, 2017. Disponible sur <https://epistemocritique.org/ entre-loeil-monde-dispositifs-dune-nouvelle-epistemologie-visuelle-sciences-de-

nature-1740-1840/>.

18. Comme le souligne Giulia Ferro-Milone dans les notes de sa traduction italienne de Le Petit Zachée (Fiabe, éd. citée), la référence à l'odeur particulière du cinabre est présente dans les dictionnaires de l'époque comme le Grammatisch-kritisches Worterbuch de Johann Christoph Adelung (1774-1786).

19. Il faut souligner que dans le sous-groupe des sept contes de fées du corpus hoffmannien, l'utilisation de personnages et de situations inquiétantes est extrêmement réduite, atténuée par une fin heureuse ou même absente (c'est le cas du Petit Zachée).

20. E.T.A. Hoffmann, Le Petit Zachée surnommé Cinabre, éd. citée, p. 134.

21. Hoffmann prend le pot de chambre comme objet caractéristique d'un mélange d'écriture ironique et de genre féerique presque certainement à partir de Wieland qui, dans Les Aventures merveilleuses de Don Sylvio de Rosalva de 1764, attribue au prince Biribinker plusieurs amours dont un avec une sylphide transformée en pot de chambre. À son tour, Wieland prend le motif du pot à partir du Sultan Misapouf et la princesse Grisemine de l'abbé de Voisenon, écrit quelques années plus tôt, ce qui nous permet de déceler le dense réseau d'influences entre la France et l'Allemagne en ce qui concerne les thèmes et les motifs du merveilleux (parmi ceux-ci également la sylphide qui reviendra dans Hoffmann, mais de manière tangentielle).

22. "Ses petits yeux étaient clos, son petit nez livide et sa bouche faiblement contractée en un léger sourire ; quant à ses cheveux, d'un brun foncé, ils retombaient autour de sa tête en boucles magnifiques. La chanoinesse les caressa ; et une ligne rouge brilla aussitôt d'une faible lueur. » (E.T.A. Hoffmann, Le Petit Zachée surnommé Cinabre, éd. citée, p. 133).

23. Les pages dans lesquelles on raconte comment les Lumières ont chassé les fées de la région sont parmi les plus ironiques de la réédition citée. Sur la critique hoffmannienne de l'utilitarisme scientifique dans Le Petit Zachée, voir É. Achermann, « Klein Zaches genannt Zinnober. Ein Märchen (1819) », dans D. Kremer (dir.), E.T.A. Hoffmann. Leben - Werk - Wirkung, Berlin, De Gruyter, 2012, p. 215-224.

24. Le passage du merveilleux au quotidien implique également un changement de langue : de l'italien (Rosabelverde) à l'allemand (Rosenschön). Pour Hoffmann, l'Italie constitue un lieu 
d'imagination jamais soumis à l'épreuve de la réalité (sa correspondance avec Hippel montre clairement son projet de voyage en Italie via la Silésie et Vienne, projet qui a été reporté à plusieurs reprises et qui a finalement échoué à cause des guerres napoléoniennes). L'Italie, peutêtre en raison de sa nature d'horizon jamais atteint, assume toujours dans les textes hoffmanniens les couleurs d'un lieu exotique, métamorphique, ouvert à la variation infinie des mélanges entre réel et imaginaire. Si les démons et les magiciens les plus réussis de ses contes portent des noms italiens (Coppola, Dappertutto, Celionati pour n'en citer que quelques-uns), Rome se révèle un véritable moteur de production de merveille dans le conte Princesse Brambilla. Sur la perspective avec laquelle Hoffmann regarde l'Italie, voir S. Acciaioli, «Italia anelata, Italia straniata, Italia ironizzata. E.T.A. Hoffmann e Wilhelm Hauf», LEA - Lingue e letterature d'Oriente e d'Occidente, $\mathrm{n}^{\circ} 2$, 2013, p. 403-422, et S. M. Moraldo (dir.), Das Land der Sehnsucht. E.T.A. Hoffmann und Italien, Heidelberg, Winter, 2002.

25. E.T.A. Hoffmann, Klein Zaches genannt Zinnober, éd. citée, p. 541.

26. Ibid., p. 584.

27. Ibid., p. 590.

28. E.T.A. Hoffmann, Le Petit Zachée surnommé Cinabre, éd. citée, p. 83.

29. Ibid., p. 98.

30. Der goldne Topf fait partie du deuxième volume (Band 2/1: Fantasiestücke in Callott's Manier. Werke 1814) de l'édition intégrale des œuvres d'E.T.A. Hoffmann (voir note 1) à laquelle on fait référence pour les citations en allemand du texte.

31. E.T.A. Hoffmann, Le Vase d'or, éd. citée, p. 15-16.

32. Sur la réception allemande des théories de Louis-Bertrand Castel, voir M. Franssen, "The ocular Harpsichord of Louis-Bertrand Castel. The Science and Aesthetics of an Eighteenth-Century Cause Célèbre ", Tractrix - Yearbook for the history of science, medicine, technology and mathematics, vol. 3, 1991, p. 15-77. Voir aussi T. L. Hankins, «The Ocular Harpsichord of Louis-Bertrand Castel; Or, The Instrument That Wasn't » Osiris, vol. 9 (Instruments), 1994, p. 141-156.

33. Le clavecin oculaire de Louis-Bertrand Castel a été introduit en Allemagne par Georg-Philipp Telemann, qui a rencontré Castel lors de son voyage à Paris et à qui il a consacré son livre Beschreibung der Augenorgel de 1739. Le système de Castel, notamment avec la réimpression du texte de Telemann en 1742 dans la Musikalischer Bibliothek de Lorenz Christoph Mizler, sera fortement critiqué par la Societät der Musicalischen Wissenschaften. Il est probable qu'Hoffmann ait pris connaissance du clavecin oculaire grâce aux remarques polémiques d'Ernst Ludwig Gerber dans son Tonkünstler-Lexicon de 1790.

34. E.T.A. Hoffmann, Ein Brief des Kapellmeisters Johannes Kreisler, dans E.T.A. Hoffmann, Sämtliche Werke in sechs Bänden, Band 3: Nachtstücke/Klein Zaches/Prinzessin Brambilla. Werke 1816-1820, éd. citée, p. 662 (ma traduction).

35. E.T.A. Hoffmann, Le Vase d'or, éd. citée, p. 19.

36. Ibid., p. 63.

37. J. W. Goethe, Zur Farbenlehre [1810], Berlin, Hofenberg, 2016, vol. 1, sect. 2. Pour l'édition française : Traité des couleurs, Laboissière-en-Thelle, Triades, 2017.

38. Selon les contes, la fleur pourpre est tantôt un lis, tantôt une amaryllis, tantôt une rose. En particulier, dans Le Vase d'or il s'agit d'une amaryllis, dans Le Petit Zachée d'une rose.

39. Sur la poétique de la fleur bleue chez Novalis et sur l'importance du bleu dans la littérature allemande, voir A. Valtolina, Blu e poesia, Milan, Bruno Mondadori, 2002. Pour un regard plus général sur les couleurs dans le romantisme, voir W. Pape (dir.), Die Farben der Romantik: Physik und Physiologie, Kunst und Literatur, Berlin, De Gruyter, 2014.

40. J. W. Goethe, Traité des couleurs, éd. fr. citée, p. 271. Dans le texte allemand: «Wer die prismatische Entstehung des Purpurs kennt, der wird nicht paradox finden, wenn wir behaupten, daß diese Farbe teils actu, teils potentia alle andern Farben enthalte.» (J.W. Goethe, Zur Farbenlehre [1810], Berlin, Zenodot Verlagsgesellscha, [édition Kindle], 2016, p. 180). 
41. Sur le concept de choc perceptif au sein de la culture médicale et pseudo-médicale entre les $\mathrm{XVIII}^{\mathrm{e}}$ et XIX ${ }^{\mathrm{e}}$ siècles, je renvoie à mon étude : "Pseudopodes, carapaces et projectiles. Imaginaire de la sensibilité et dispositifs de captation attentionnelle entre les XVIII ${ }^{\mathrm{e}}$ et XIX ${ }^{\mathrm{e}}$ siècles ", dans Y. Citton et E. Doudet (dir.), Écologies de l'attention et archéologie des media, Grenoble, UGA Éditions, 2019, p. 159-174.

42. E.T.A. Hoffmann, L'Homme au sable, éd. citée, p. 106.

43. Sur la relation entre Hoffmann et le magnétisme, voir J. Barkhoff, «Geschechterantropologie und Mesmerismus-Literarische Magnetiseurinnen bei und um E.T.A. Hoffmann ", dans G. Neumann (dir.), ,Hoffmaneske Geschichte'. Zu einer Literaturwissenschaft als Kulturwissenschaft, Würzburg, Königshausen \& Neumann, 2005, p. 15-42.

44. En cela, Hoffmann poursuit un discours d'expérimentation littéraire déjà en cours dans l'univers des contes de fées du milieu du XVIII siècle. Sur ce sujet, voir A. Gaillard, «Les contes, un laboratoire des sens: augmentation sensorielle, polysensorialité et synesthésies ", Féeries, $\mathrm{n}^{\circ} 15$ (L'univers sensible des contes), 2018, p. 1-12.

\section{RÉSUMÉS}

Cette étude comparée des contes Le Petit Zachée surnommé Cinabre et Le Vase d'or vise à montrer les mécanismes sensoriels de l'expérience merveilleuse chez E.T.A. Hoffmann. En particulier, la couleur semble avoir un rôle décisif dans les stratégies de dérèglement perceptif qui accompagnent le passage du réel à une dimension surnaturelle d'enchantement poétique.

This comparative study of the tales Little Zaches Called Cinnabar and The Golden Vase aims to show the sensory mechanisms of the wonderful experience in E.T.A. Hoffmann. In particular, color seems to have a decisive role in the strategies of perceptual disruption that accompany the passage from reality to a supernatural dimension of poetic enchantement.

\section{INDEX}

Keywords : E.T.A. Hoffmann, Kunstmärchen, marvelous, colors theory, synaesthesia

Mots-clés : E.T.A. Hoffmann, Kunstmärchen, merveilleux, théorie des couleurs, synesthésie

\section{AUTEUR}

\section{ISABELLA MATTAZZI}

Université de Ferrare

isabella.mattazzi@unife.it 